\title{
Structure, Diffusion, and Permeability of Protein-Stabilized Monodispersed Oil in Water Emulsions and Their Gels: A Self-Diffusion NMR Study
}

\author{
Alexandre I. Romoscanu, ${ }^{\dagger}$ Annia Fenollosa,${ }^{\dagger}$ Simone Acquistapace, ${ }^{\dagger}$ Deniz Gunes, ${ }^{\dagger}$ \\ Teresa Martins-Deuchande, ${ }^{\dagger}$ Pascal Clausen, ${ }^{\dagger}$ Raffaele Mezzenga, ${ }^{*}$ Magnus Nydén, ${ }^{\S}$ Klaus Zick, \\ and Eric Hughes* ${ }^{* \dagger}$ \\ ${ }^{\dagger}$ Nestle Research Centre, NESTEC LTD, 1000 Lausanne 26, Switzerland, ${ }^{\star}$ Department of Physics and Fribourg \\ Center for Nanomaterials, University of Fribourg, Fribourg, Switzerland, ${ }^{\S}$ Department of Chemical and Biological \\ Engineering, Chalmers University of Technology, Gothenbourg SE-412 96, Sweden, and "Bruker BioSpin GmbH,
} Silberstreifen, 76287 Rheinstetten, Germany

\begin{abstract}
Self-diffusion NMR is used to investigate monodispersed oil in water emulsions and the subsequent gel formed by removing the water through evaporation. The radius of the oil droplets in the emulsions is measured using a number of diffusion methods based on the measurement of the mean squared displacement of the oil, water, and tracer molecules. The results are consistent with the known size of the emulsions. Bragg-like reflections due to the restricted diffusion of the water around the oil droplets are observed due to the low polydispersity of the emulsions and the dense packing. The resulting data are fitted to a pore glass model to give the diameter of both the pools of interstitial water and the oil droplets. In the gel, information on the residual three-dimensional structure is obtained using the short time behavior of the effective diffusion coefficient to give the surface to volume ratio of the residual protein network structure. The values for the surface to volume ratio are found to be consistent with the expected increase of the surface area of monodisperse droplets forming a gel network. At long diffusion observation times, the permeability of the network structure is investigated by diffusion NMR to give a complete picture of the colloidal system considered.
\end{abstract}

\section{Introduction}

Microfluidic techniques hold a lot of promise for the preparation of new materials with interesting properties. ${ }^{1-3}$ The techniques also offer a means of preparing materials in a controlled and reproducible manner so that their physical properties can be conveniently studied. ${ }^{4}$ This approach should be particularly useful for studying processed food materials. In processed foods, the physical properties relevant to the function and structure of the food matrix are often hidden by the heterogeneity of the food molecules themselves. ${ }^{5}$ Furthermore, in the preparation of food materials such as foams and emulsions, fine control over the manufacturing process is typically very limited.

In this paper, we demonstrate that improved control over the preparation process can be achieved by applying microfluidic techniques to model food materials, for example, protein stabilized oil in water emulsions. By using the concepts of microfluidics, stable monodispersed emulsions were made with controlled droplet diameters. The physical properties of the emulsions (size, permeability of the interface, and stability) are then investigated by pulsed-field gradient NMR self-diffusion techniques. High internal phase emulsions (HIPEs) can be produced by crosslinking the protein stabilized interface via thermal, enzymatic, or chemical processes followed by slow evaporation of the water.

*To whom correspondence should be addressed. E-mail: eric.hughes@ rdls.nestle.com.

(1) Xu, S.; Nie, Z.; Seo, M.; Lewis, P.; Kumacheva, E.; Stone, H. A.; Garstecki, P.; Weibel, D. B.; Gitlin, I.; Whitesides, G. M. Angew. Chem., Int. Ed 2005, 44, $724-728$.

(2) Whitesides, G. M. Nature 2006, 442, 368-373.

(3) Squires, T. M.; Quake, S. R. Rev. Mod. Phys. 2005, 77, 977-1026.

(4) Woodward, A.; Cosgrove, T.; Espidel, J.; Jenkins, P.; Shaw, N. Soft Matter 2007, 3, 627-633.

(5) Mezzenga, R.; Schurtenberger, P.; Burbidge, A.; Michel, M. Nat. Mater. $\mathbf{2 0 0 5}, 4,729-740$.
The residual water content can be decreased virtually to zero, while preserving the three-dimensional structures of the oil in water emulsion. ${ }^{6,7}$ These systems ultimately resemble protein in oil gels. A schematic of how the gels are created and their typical physical properties is given in Figure 1.

The diffusion and permeation properties of the gel are examined in the present work by self-diffusion NMR. It is shown that, by controlling the preparation of the emulsion, in particular the polydispersity of the droplet size, via microfluidic techniques, a much more detailed and unambiguous analysis can be made of the physical properties of the emulsion and the resulting gel without resorting to assumptions about the size distribution of the emulsion or gel.

\section{Theoretical Background}

Pulsed-field gradient techniques have been used to measure the self-diffusion coefficient of many systems in which the molecules are free to diffuse without restriction or hindrance. The normalized NMR echo signal, $S / S_{0}(g, \Delta)$, as a function of the diffusion observation time, $\Delta$, and gradient strength, $g$, for a self-diffusion experiment such as the pulsed-field gradient stimulated echo (PGSTE) self-diffusion sequence, as introduced by Tanner ${ }^{9}$ and shown in Figure 2, is given by the following equation.

$$
\frac{S}{S_{0}}(g, \Delta)=\exp \left(-(\gamma g \delta)^{2} D\left(\Delta-\frac{\delta}{3}\right)\right)
$$

\footnotetext{
(6) Romoscanu, A. I.; Mezzenga, R. Langmuir 2006, 22, 7812-7818.

(7) Romoscanu, A. I.; Mezzenga, R. Langmuir 2005, 21, 9689-9697.

(8) Canilho, N.; Kasëini, E.; Schlüter, A. D.; Ruokolainen, J.; Mezzenga, R. Macromolecules 2007, 40, 7609-7616.
}

(9) Tanner, J. E. J. Chem. Phys. 1970, 52, 2523-2526. 

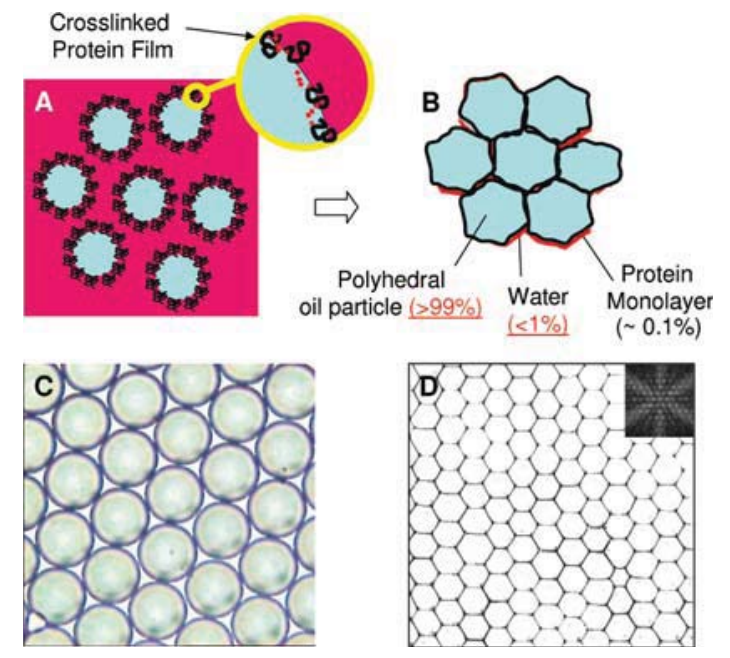

Figure 1. Sketch of the procedure leading to densely packed monodisperse and cross-linked oil in water emulsions and subsequent gels: (A) protein-stabilized oil in water emulsions with crosslinked protein interface, (B) resulting gel after removal of water, (C) optical image of $30 \mu \mathrm{m}$ oil droplets prior to water evaporation, and (D) optical image of same oil droplets as in (C) after water evaporation. The inset in (D) gives the fast Fourier transform (FFT), showing a typical hexagonal diffraction pattern with q1:q2: $\mathrm{q} 3: \mathrm{q} 4$ reflections in the reciprocal q lattice spaced as $1: \sqrt{ } 3: 2: \sqrt{ } 7 .{ }^{8}$

where $\Delta$, the diffusion observation time, is defined as the separation between the two gradient pulses, measured from their leading edges, $\delta$ is the duration of the gradient pulse, $g$ is the strength of the gradient pulse, $\gamma$ is the gyromagnetic ratio, $S$ is the magnitude of the NMR echo signal as a function of increased gradient strength, and $S_{0}$ is the NMR signal in the absence of gradient pulses. $D$ is the self-diffusion coefficient of the liquid.

In the presence of hindered or restricted diffusion, where the mean squared displacement, $\left\langle z^{2}\right\rangle$, of molecules is influenced by boundary interfaces as for fluids in porous media, or the continuous and dispersed phases of an emulsion, then it is no longer strictly correct to apply the above equation to the echo decay of a pulsed-field gradient NMR experiment in order to obtain the free self-diffusion coefficient. The pulsed-field gradient NMR experiment when applied to such systems is still useful because it can provide information on the structure of the restricting geometry. There are broadly four main approaches by which the experiment can provide information on the restricting geometry. The first is to determine the propagator for the diffusion in the restricted environment and derive a new equation for the relationship between the echo signal, the diffusion coefficient, and the restricting geometry. This approach has only been achieved for very simple geometries, a sphere being one of them. A solution for the echo decay within in a sphere has been derived by Murday and Cotts $^{10}$ and is given by the following equation:

$$
\begin{gathered}
\ln \frac{S(2 \Delta, \delta, g)}{S_{0}}=\frac{2 \Delta}{T_{2}}-2 \gamma^{2} g^{2} \sum_{m=1}^{\infty}\left[\alpha_{m}^{2}\left(\alpha_{m}^{2} r^{2}-2\right)\right]^{-1} \\
\quad \times\left(\frac{2 \delta}{\alpha_{m}^{2} D}-\left(2+\exp \left[-\alpha_{m}^{2} D(\Delta-\delta)\right]\right.\right. \\
-2 \exp \left(-\alpha_{m}^{2} D \delta\right)-2 \exp \left(-\alpha_{m}^{2} D \Delta\right) \\
\left.\left.+\exp \left[-\alpha_{m}^{2} D(\Delta+\delta)\right]\right) /\left(\alpha_{m}^{2} D\right)^{2}\right)
\end{gathered}
$$

where the echo signal depends solely on the free self-diffusion coefficient of the confined liquid, $D$, the radius of the sphere, $r$, and the particular parameters of the experiment, $\delta, \Delta$, and $g . \alpha_{m}$ is the $m$ th root of the Bessel equation $(1 / \alpha r) J_{3 / 2}(\operatorname{ar})=J_{5 / 2}(\alpha r)$. This approach has found use in a number of studies, ${ }^{11,12}$ especially in the area of emulsions where it is used in conjunction with a distribution function to obtain the average diameter of the droplets and the width of the distribution. Typically, for emulsions, a log-normal distribution has been assumed. ${ }^{13}$

At long diffusion observation times, the mean squared displacement of the oil molecules, $\left\langle z^{2}\right\rangle$ will be limited by the reflecting boundaries of the spherical droplets in the emulsion. The meansquared displacement in one dimension is related to the free diffusion coefficient by the following equation.

$$
\left\langle z^{2}\right\rangle=2 D \Delta
$$

and it has been shown that, independent of restricting geometry, $\left\langle z^{2}\right\rangle$ can always be obtained directly from the initial slope of the echo decay as long as the gradient strength is kept small. ${ }^{14}$ Typically, the maximum gradient is set to a value that reduces the echo intensity by a factor of 0.7 .

$$
\frac{S}{S_{0}} \approx 1-\frac{1}{2} \gamma^{2} g^{2} \delta^{2}\left\langle z^{2}\right\rangle
$$

and for a sphere of radius $r$,

$$
\left\langle z^{2}\right\rangle=\frac{2 r^{2}}{5}
$$

Therefore, if the diffusion experiment can be performed at sufficiently long diffusion observation times such that all of the molecules within the droplets have encountered the walls of the restriction many times and there is no component of free diffusion, then a value for the radius of the droplets can be obtained. An estimation of the required observation time can be obtained from knowing the free diffusion coefficient of the internal phase and utilizing the following inequality $2 D \Delta=\left\langle z^{2}\right\rangle \gg r^{2}$.

At very short diffusion observation times, $\Delta$, very few molecules within the confining geometry will have encountered the restricting walls. Therefore, an apparent diffusion coefficient $D_{\text {app }}$ can be calculated using directly eq 1 or eqs 4 and 3 which will be very close to the free self-diffusion coefficient of the liquid, $D$, but slightly reduced due to the influence of hindered diffusion of the molecules close to the confining surface. As the diffusion observation time is increased, the confinement of the walls increasingly influences the apparent diffusion coefficient so that it deviates further and further away from the free diffusion value. Mitra and co-workers ${ }^{15}$ have shown that this reduction, to first order, is linearly proportional to $(D \Delta)^{1 / 2}$ and the slope is proportional to the surface to volume ratio of the confining geometry. They give the following relationship for the apparent diffusion coefficient:

$$
\frac{D_{\text {app }}}{D_{0}}=1-\frac{4}{9 \sqrt{\pi}} \frac{S}{V}\left(D_{0} \Delta\right)^{1 / 2}+O\left(D_{0} \Delta\right)
$$

(11) Denkova, P. S.; Tcholakova, S.; Denkov, N. D.; Danov, K. D.; Campbell, B.; Shawl, C.; Kim, D. Langmuir 2004, 20, 11402-11413.

(12) Balinov, B.; Söderman, O.; Wärnheim, T. J. Am. Oil Chem. Soc. 1994, 71, 513-518.

(13) Packer, K. J.; Rees, C. J. Colloid Interface Sci. 1972, 40, 206-218.

(14) Callaghan, P. T. Principles of Nuclear Magnetic Resonance Microscopy; Clarenden Press: Oxford, UK, 1991.

(15) Mitra, P. P.; Sen, P. N.; Schwartz, L. M.; Doussal, P. L. Phys. Rev. Lett. 1992, 68, 3555-3558. 


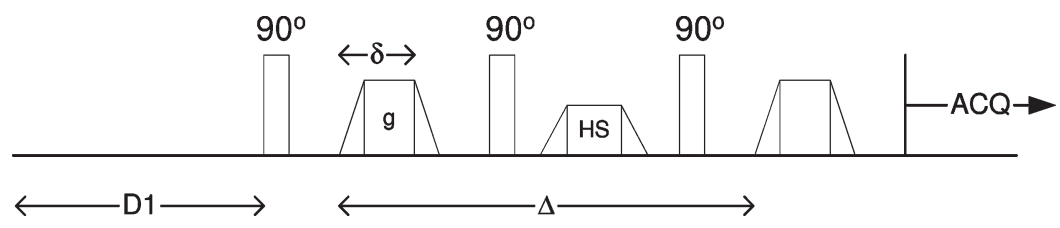

Figure 2. Pulsed-field stimulated echo pulse sequence using trapezoidal gradients. $\Delta$ is the diffusion observation time, measured from the leading edges of the two gradient pulses. $\delta$ is the duration of the gradient pulse. HS is a gradient homospoil pulse. D1 is the recycle delay of the experiment. All other pulses are radio frequency pulses.

for a sphere, $S / V=3 / r$; therefore, for the emulsion droplets, the size can be calculated from the initial slope. The relationship in eq 6 is valid for any type of geometry and for both impermeable and permeable structures. ${ }^{16}$

Finally, Callaghan and co-workers ${ }^{17}$ have shown that "diffraction-like" behavior can be observed during the PGSTE experiment on systems such as water around randomly packed monodispersed spheres. The NMR signal attenuation of a pulsed gradient diffusion experiment, under certain conditions of gradient strength and diffusion observation time, show maxima in the signal decay as a function of increasing gradient strength. The echo decay has been directly related to the water pore separation length between the packed spheres using a model based on a pore glass description, ${ }^{18}$ where the pore spacing varies and there is orientational randomness. The echo intensity, $S$, as a function of the diffusion observation time, $\Delta$, and the magnitude of the reciprocal space vector, $q$, where $q=\gamma g \delta / 2 \pi$, is then given by the following two equations:

$$
S(q, \Delta)=\left|S_{0}(q)\right|^{2} \exp \left[-\frac{6 D_{\text {eff }} \Delta}{b^{2}+3 \xi^{2}}\left(1-\exp \left(-2 \pi^{2} q^{2} \xi^{2}\right) \frac{\sin (2 \pi q b)}{2 \pi q b}\right)\right]
$$

$$
\left|S_{0}(q)\right|^{2}=\left|\frac{3[2 \pi q a \cos (2 \pi q a)-\sin (2 \pi q a)]}{(2 \pi q a)^{3}}\right|^{2}
$$

where $b$ is the mean pore water spacing and $\xi$ is the standard deviation based on a Gaussian distribution. $D_{\text {eff }}$ is the long-range diffusion coefficient for Brownian motion through the permeable structure. $\left|S_{0}(q)\right|^{2}$ is the form factor of the pores, considered as spheres with a diameter of $a$.

Söderman and co-workers ${ }^{19}$ have demonstrated similar effects for concentrated water in oil emulsions, relating the first and second maxima to certain characteristic distances of the deformed droplets using a simple two-dimensional hexagonal model. In the oil in water emulsions used in this study, similar effects should be observable for the water surrounding the oil droplets to yield an estimate of the oil droplet diameter.

The permeability of the gel system can be investigated by observing the apparent diffusion coefficient at long diffusion observation times. ${ }^{20}$ The reduced permeability, $P$, is related to the apparent diffusion at long diffusion observation times by the following equation:

$$
\frac{D_{0}}{D_{\infty}}=1+\frac{1}{P}
$$

(16) Sen, P. N.; Schwartz, L. M.; Mitra, P. P. Magn. Reson. Imaging 1994, 12 , 227-230.

(17) Callaghan, P. T.; Coy, A.; MacGowan, D.; Packer, K. J.; Zelaya, F. O. Nature 1991, 351, 467-469.

(18) Callaghan, P. T.; Coy, A.; Halpin, T. P. J.; MacGowan, D.; Packer, K. J.; Zelaya, F. O. J. Chem. Phys. 1992, 97, 651-662

(19) Håkansson, B.; Pons, R.; Söderman, O. Langmuir 1999, 15, 988-991.

(20) Tanner, J. E. J. Chem. Phys. 1978, 69, 1748-1754. where $P=a p / D_{0}$, with $a$ being the barrier separation and $p$ the permeability. For the barrier separation value, $a$, of the gel, the diameter of the precursor emulsion can be used. If very small values of $D_{\infty} / D_{0}$ are observed for the gel system, then the permeability can be approximated by the following equation:

$$
p=\frac{D_{\infty}}{D_{0}} \frac{D_{0}}{a}=\frac{D_{\infty}}{a}
$$

Latour and co-workers ${ }^{21,22}$ have shown that, for porous and permeable material, the short-term behavior of $D_{\text {app }}$ as given by eq 6 and the long time behavior of $D_{\text {app }}$ as given by

$$
\frac{D_{\infty}}{D_{0}}=\frac{1}{\alpha}
$$

where $\alpha$ is the tortuosity of the pore space, can be linked through a two point Padé approximation by the following formula.

$$
\frac{D_{\text {app }}}{D_{0}}=1-\left(1-\frac{1}{\alpha}\right) \frac{\frac{4}{9 \sqrt{\pi}} \frac{S}{V} \sqrt{D_{0} \Delta}+\left(1-\frac{1}{\alpha}\right) \frac{\Delta}{\theta}}{\left(1-\frac{1}{\alpha}\right)+\frac{4}{9 \sqrt{\pi}} \frac{S}{V} \sqrt{D_{0} \Delta}+\left(1-\frac{1}{\alpha}\right) \frac{\Delta}{\theta}}
$$

where $\theta$ has the dimensions of time. If $D_{\infty}$ cannot be obtained due to limitations of relaxation, then eq $12 \mathrm{can}$ be used to fit the data and obtain a value for the tortuosity and then through eqs 11 and 10 an estimate of the permeability of the gel can be made.

\section{Experimental Section}

Materials. $\beta$-Lactoglobulin $(\beta$ - $\mathrm{Lg})$ was supplied by Davisco Foods Inc. (Eden Prairie, MN), under batch number JE 2633420. $\beta$ - $\mathrm{Lg}$ is a globular protein with molecular weight of $18 \mathrm{kDa}$. The protein used contained $97 \%$ protein on dry basis (of which $92 \%$ is $\beta$ - $\mathrm{Lg}$ ), ca. $2.5 \%$ ash, and $0.5 \%$ lactose. The protein was used as received without further purification in the form of a $0.1 \mathrm{wt} \%$ protein solution in $20 \mathrm{mM}$ imidazole buffer adjusted to $\mathrm{pH}=7.0$ with $1 \mathrm{M} \mathrm{NaOH}$. Medium chain triglyceride (MCT, supplied by Cognis, Germany) was used as the dispersed oil phase. Hexamethyldisilane (HMD) at a concentration of $1 \mathrm{wt} \%$ was used in the oil phase as a tracer molecule in some of the emulsion and subsequent gel systems. As a protein cross-linking agent, glutaraldehyde was used, supplied by Fluka (Switzerland) as a $5.6 \mathrm{M}$ (50 wt \%) solution. Monodisperse polystyrene beads of diameter $30 \mu \mathrm{m}$ dispersed in a dilute aqueous medium were supplied by Duke Scientific (cat. no.: 4230A). They were used as a standard to compare with experiments performed on emulsions of similar size.

Preparation of the Monodispersed Emulsions. Monodispersed oil in water emulsions were prepared using the coflowing

(21) Latour, L. L.; Mitra, P. P.; Kleinberg, R. L.; Sotak, C. H. J. Magn. Reson., Ser. A $1993,101,342-346$.

(22) Latour, L. L.; Karel Svoboda, P. P. M.; Sotak, C. H. Proc. Natl. Acad. Sci. U.S.A. 1994, 91, 1229-1233. 
stream drop break-off method published by Umbanhowar et al. ${ }^{23}$ In this method, pressure-driven drops form at the tip of a static capillary dipped into a flowing aqueous phase loaded with a suitable surfactant. Upon reaching a size where the drag due to the coflowing liquid exceeds the interfacial tension, the droplet breaks off from the end of the tip. The uniformity of the conditions at the end of the tip lead to the formation of a low polydisperse emulsion.

In the present arrangement, the most accessible droplet sizeregulating parameters are the relative velocity between flow and tip and the capillary diameter. By varying these parameters, we were able to produce low polydisperse emulsions with droplet diameters ranging from ca. 20 to $100 \mu \mathrm{m}$ at a maximum rate of ca. $1 \mathrm{~mL}$ of dispersed phase per hour. Both homemade and commercial capillaries (Eppendorf AG, Hamburg, Germany) were used with typical tip diameters of 150 and $15 \mu \mathrm{m}$, respectively. The rotating cup that held the aqueous phase had an inner diameter of $8 \mathrm{~cm}$ and height of $6 \mathrm{~cm}$. The cup was rotated at an angular velocity of $442(2 \pi) / \mathrm{min}$.

The unabsorbed protein acting as the surfactant in the aqueous phase was removed by allowing the emulsion to cream in a decantation flask and replacing the protein aqueous phase with just imidazole buffer at $\mathrm{pH}=7.020 \mathrm{mM}$. The irreversibility of the protein adsorption to the interface allows the preparation of stable emulsions with vanishingly low amounts of unadsorbed protein. Finally, for emulsions that were used to form a gel, the adsorbed protein molecules were cross-linked to ensure protein layer stability upon the removal of the continuous phase. This was achieved by pouring the monodisperse emulsion into a $1 \mathrm{wt} \% \mathrm{pH} 7.020 \mathrm{mM}$ imidazole buffered glutaraldehyde solution for $1 \mathrm{~h}$.

The samples were prepared for NMR analysis in the following manner: the oil in water emulsion was concentrated using a Shigemi NMR tube (Shigemi Inc., Allison Park, PA). The dilute emulsion was pipetted into the Shigemi tube, and the plunger was inserted into the tube. The tube was then placed upside down so that the oil droplets would float to the bottom of the tube. After 1 day, the excess water was removed from the Shigemi tube by slowly inserting the plunger down the tube and expelling the water until only the oil droplets and the interstitial water remained. A similar procedure was used to prepare a sample of the polystyrene beads for NMR analysis. In order to make the gel in the NMR tube, a dilute emulsion was placed in the tube and the water was slowly evaporated away to make a clear gel by flowing nitrogen gas over the surface of the sample. Romoscanu and Mezzenga ${ }^{7}$ give further details of the preparation methods for the emulsions and resulting high internal phase emulsion (HIPE) gels.

NMR. The NMR experiments were performed on a Bruker AVANCE DSX400 wide-bore NMR spectrometer. The Larmor frequency for protons was $400.13 \mathrm{MHz}$. Two self-diffusion probes were used for the self-diffusion experiments, a Bruker DIFF30 probe and a Bruker DIFF25 probe. The DIFF25 used a home-built $5 \mathrm{~mm}$ diameter single tuned proton coil constructed in the group of Dr. Magnus Nyden (Chalmers University, Gothenburg). The proton $90^{\circ}$ pulse was 8.5 and $10 \mu$ s for the DIFF30 and DIFF25 probes, respectively. A Bruker GREAT60 gradient amplifier was used with the DIFF30 probe and a Bruker BAFPA 40 gradient amplifier was used with the DIFF25 probe. The temperature was set to $25.0^{\circ} \mathrm{C}$ and was calibrated using a standard ethylene glycol sample (Bruker Biospin). The temperature was maintained using the gradient cooling water system flowing through the gradient system of the probe. The gradient amplifiers were calibrated using a doped $1 \%$ water in $\mathrm{D}_{2} \mathrm{O}$ sample (Bruker Biospin) to give a self-diffusion coefficient for the water of $1.90 \times 10^{-9} \mathrm{~m}^{2} \mathrm{~s}^{-1} .^{24}$ The pulsed-gradient stimulated echo ${ }^{9}$ (PGSTE) pulse sequence was used for the diffusion experiments employing trapezoidal shaped gradient pulses. The conditions for

(23) Umbanhowar, P. B.; Prasad, V.; Weitz, D. A. Langmuir 2000, 16, 347-351. (24) Holz, M.; Weingärtner, H. J. Magn. Reson. 1991, 92, 115-125. the calibration experiment were a gradient pulse of $1.0 \mathrm{~ms}$, a diffusion delay of $20 \mathrm{~ms}$, and a recycle delay of $2 \mathrm{~s}$. An eight step phase cycle was used. Eight scans were taken for each gradient increment, and 16 gradient steps were employed.

The time dependence of the apparent self-diffusion coefficients of the oil and hexamethyldisilane were measured in both the concentrated emulsion and the dried emulsion over a diffusion observation time range of $20 \mathrm{~ms}$ to $8 \mathrm{~s}$. The duration of the pulse gradient was set to $1.0 \mathrm{~ms}$, and the slope time of the trapezoidal gradient pulse was $0.3 \mathrm{~ms}$. The delay between the first two $90^{\circ}$ pulses was set to the minimum and was calculated by the spectrometer software and varied between 2.62 and $2.82 \mathrm{~ms}$ depending on the size of the gradient pulse. The stabilization time after the gradient pulse was set to $1.0 \mathrm{~ms}$. The maximum gradient strength for each diffusion observation time was adjusted so that the signal decay was reduced to 0.7 of its original value. Eight gradient increments were used for each experiment, and depending on the signal-to-noise ratio 8 or 64 transients were acquired at each gradient step. The recycle delay was kept constant at $15 \mathrm{~s}$ for the emulsion sample and $10 \mathrm{~s}$ for the gel. For the diffusion experiments to measure the surface to volume ratio, the minimum gradient starting gradient applied was $0.2 \mathrm{G} / \mathrm{cm}$.

\section{Results and Discussion}

Microscopy and Particle Sizing. Size distribution analyses of the emulsions were performed using an AccuSizer 780-APS instrument (Particle Sizing systems, Santa Barbara, CA) in light extinction mode. The emulsion droplet distribution was characterized in terms of the mean volume diameter $d$ and the uniformity $U$ given by the two following equations: ${ }^{25}$

$$
\begin{gathered}
d=\frac{\sum_{i} N_{i} d_{i}^{4}}{\sum_{i} N_{i} d_{1}^{3}} \\
U=\frac{1}{d} \frac{\sum_{i} N_{i} d_{i}^{3}\left|\bar{d}-d_{i}\right|}{\sum_{i} N_{i} d_{i}^{3}}
\end{gathered}
$$

where $N_{i}$ is the number of droplets with a diameter $d_{i}$ and $\bar{d}$ is the median diameter.

Microscopy was performed on an instrument from Zeiss, Germany at a magnification of 10. A typical set of results for a monodisperse emulsion prepared in this study is shown in Figure 3. From the microscope image and the particle size analysis, one observes that the droplets have a narrow monomodal distribution with a median droplet diameter, $\bar{d}$, of $36.6 \mu \mathrm{m}$ and a uniformity, $U$, of $6.9 \%$. An emulsion is considered monodisperse if it has a uniformity value of less than $25 \% .{ }^{25}$ In Figure $3 c$, the results from a self-diffusion PGSTE experiment are shown for the water in the emulsion after concentrating it by creaming. At large $q$ values, maxima appear in the decaying signal that are a consequence of the restricted diffusion of the water and the uniformity of the oil droplets. The first maximum corresponds to a distance of $33.6 \mu \mathrm{m}$, close to the median value obtained for the diameter of the droplets from the particle sizing measurement.

Spin-Lattice Relaxation, $T_{1}$, in the Emulsions and Gels. One of the aims of this study was to investigate the long time diffusion properties of the oil and tracer in the emulsions and the gel systems in order to estimate the size of the emulsion droplets

(25) Schmitt, V.; Leal-Calderon, F.; Bibette, J. Top. Curr. Chem. 2003, 217, $195-215$ 

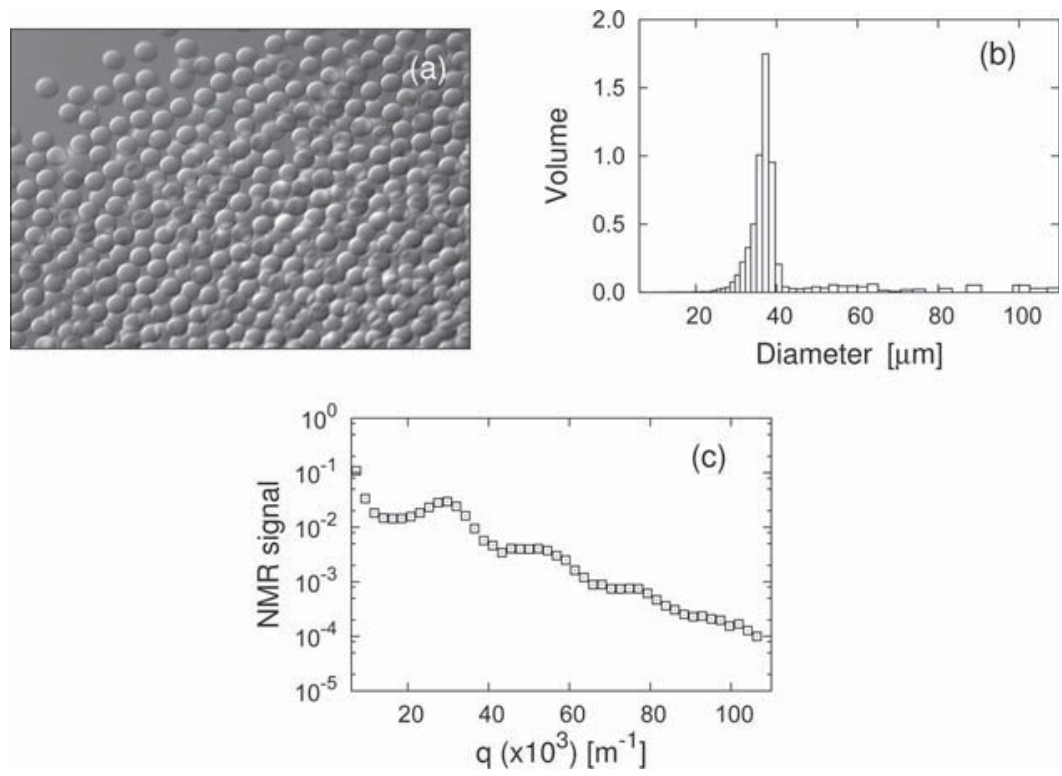

Figure 3. Analysis of oil in water emulsion made by the coflow drop-off method. (a) Microscopy image of emulsion at a magnification level of $\times 10$. (b) Particle size distribution of the emulsion giving a median droplet diameter of $36.6 \mu \mathrm{m}$ and a uniformity of $6.9 \%$. (c) Self-diffusion NMR experiment of the water in the emulsion showing diffraction peaks due to the monodisperse nature of the emulsion. The first maximum corresponds to a distance of $33.6 \mu \mathrm{m}$.

Table 1. $\mathrm{T}_{1}$ Relaxation Times in Seconds of the Water, MCT, and HMD in the Oil in Water Emulsion and $\mathrm{Gel}^{a}$

\begin{tabular}{lccc}
\hline & peak ppm & emulsion $\mathrm{T}_{1}[\mathrm{~s}]$ & gel $\mathrm{T}_{1}[\mathrm{~s}]$ \\
\hline water & 4.80 & 3.15 & \\
HMD & 0.0 & 1.94 & 2.74 \\
MCT & 2.20 & 0.52 & 0.56 \\
& 1.53 & & 0.53 \\
& 1.24 & 0.64 & 0.71 \\
& 0.84 & 1.02 & 1.13
\end{tabular}

${ }^{a}$ Only the $\mathrm{T}_{1}$ of resonances of the MCT that were baseline resolved in the two systems were measured. No water resonance is observed in the gel.

and to determine the permeability of the gel structure. Therefore, it was necessary to measure the proton spin-lattice relaxation times of the different molecules, water, MCT, and the tracer, hexamethyldisilane (HMD), in the emulsion and gel systems so that the conditions of the self-diffusion experiment could be set correctly. The spin-lattice relaxation properties of the emulsions and gels were measured by an inversion recovery experiment. The results are summarized in Table 1.

The different chemical groups of the MCT oil have various $\mathrm{T}_{1}$ relaxation rates, ranging from 0.52 to $1.13 \mathrm{~s}$. The methyl groups $(0.84 \mathrm{ppm})$ have the longest relaxation rates which are just over $1 \mathrm{~s}$. The water in the emulsion has a $\mathrm{T}_{1}$ of $3.0 \mathrm{~s}$, and the HMD has a $T_{1}$ of nearly $2.0 \mathrm{~s}$ in the emulsion and $2.7 \mathrm{~s}$ in the gel. Therefore, for the diffusion experiments, the recycle delay was set to $15 \mathrm{~s}$. In the diffusion experiments, only the methyl group was used to follow the diffusion of the oil.

Water Diffusion around Oil Droplets. From optical microscopy, the average size of the oil droplets can be estimated and an idea of the polydispersity can be attained as shown in Figure 3. The microscopy studies showed that the emulsions were of low polydispersity and that the oil droplets packed very nicely, showing some short-range order when concentrated on a microscope slide. Callaghan et al. ${ }^{17}$ first showed in a system of randomly packed monodisperse polystyrene spheres immersed in water that the response from the water to a PGSTE

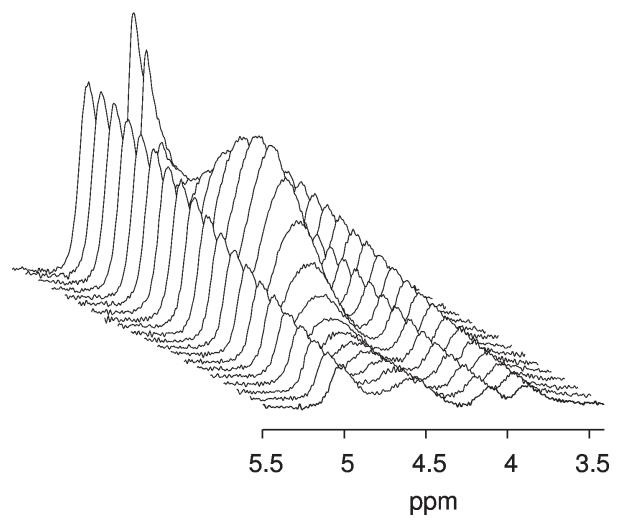

Figure 4. Diffusion response of water around concentrated monodisperse oil droplets as a function of increasing gradient strength. The spectra show good resolution of the oil and water signals. The water signal shows a maximum characteristic of diffusion around monodisperse droplets. The diameter of the oil droplets were approximately $45 \mu \mathrm{m}$. The gradient pulse was increased linearly from 12.6 to $89.4 \mathrm{G} / \mathrm{cm}$. The diffusion observation time, $\Delta$, was $600 \mathrm{~ms}$, and the gradient pulse length, $\delta$, was $1.5 \mathrm{~ms}$.

self-diffusion experiment held information on the size of the packed spheres.

Figure 4 shows the diffusion response of a typical concentrated emulsion used in this study as a function of increasing gradient strength. The figure shows an expanded view of the resonances centered around the water signal at $4.6 \mathrm{ppm}$. The resolution in the experiment is sufficient to clearly resolve the water signal from those arising from the oil. In the figure, one can observe that the water signal is no longer a single exponential decay as is the case for the oil resonances. The water response clearly shows a maximum that is due to the restricting geometry of the closely packed monodisperse oil droplets.

Figure 5 shows a more conventional representation of the nonexponential decay behavior of the water signal in two of the monodispersed emulsions used in this study. Each point is the integral of the water resonance as a function of $q$. The figure 
shows results from two emulsions of different size diameters. Plots (a) and (b) arise from the same emulsion with a droplet diameter of approximately $25 \mu \mathrm{m}$ measured under the same experimental conditions but separated in time by 2 months. Plot (c) is from an emulsion with a droplet diameter size of approximately $45 \mu \mathrm{m}$. The two different sized emulsions are distinguished by the position of the first maximum. The two plots, (a) and (b), overlap each other nearly completely, indicating that the emulsions are very stable with time. This is important, as a series of NMR diffusion experiments with good signal-to-noise ratios can sometimes take days to complete. The fact that the samples are very stable also means that they are well suited for use as model samples in the development of new NMR experiments or as test samples for the reliability and stability of the spectrometer.

The two plots, (a) and (b) in Figure 5 display two maxima occurring at $q$ values of $3.8 \times 10^{4}$ and $6.6 \times 10^{4} \mathrm{~m}^{-1}$ corresponding to a distance of $26.3 \pm 1$ and $15.2 \pm 1 \mu \mathrm{m}$, respectively. The third plot, (c), corresponding to the larger droplet diameter emulsion also shows two maxima, but the second peak is quite weak and therefore difficult to determine precisely. The positions of the two maxima for plot (c) are at $q$ values of $2.3 \times 10^{4}$ and $4.0 \times 10^{4} \mathrm{~m}^{-1}$ to give distances of $43.5 \pm 2$ and $25.0 \pm 2 \mu \mathrm{m}$, respectively. In previous work by Söderman et al. ${ }^{19}$ on a highly concentrated water in oil emulsion, similar maxima were observed for the water resonance. In that study, a $\sqrt{ } 3$ relationship was noted between the positions of the two maxima. This ratio was interpreted as a characteristic distance of the system when the spherical water droplets were modeled using a two-dimensional

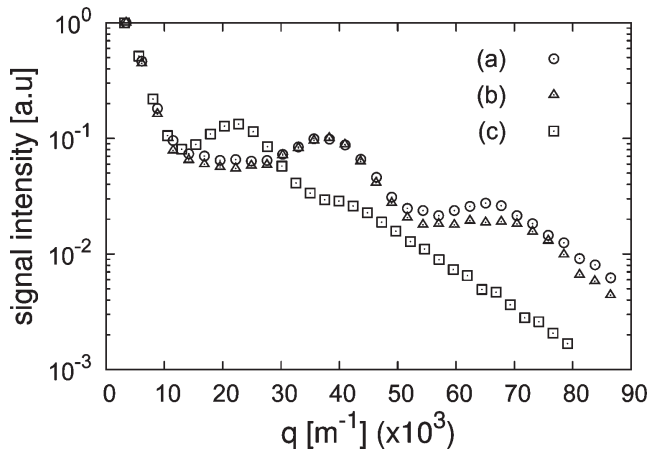

Figure 5. Comparison of water diffusion around oil droplets for two concentrated monodisperse oil in water emulsions of different size. Plots (a) and (b) are from an emulsion with a diameter of around $25 \mu \mathrm{m}$ acquired with a separation in time of 2 months. Plot (c) is another emulsion with a diameter around $45 \mu \mathrm{m}$. For the experiments, the diffusion observation time, $\Delta$, was $600 \mathrm{~ms}$ and the gradient pulse length, $\delta$, was $1.5 \mathrm{~ms}$.

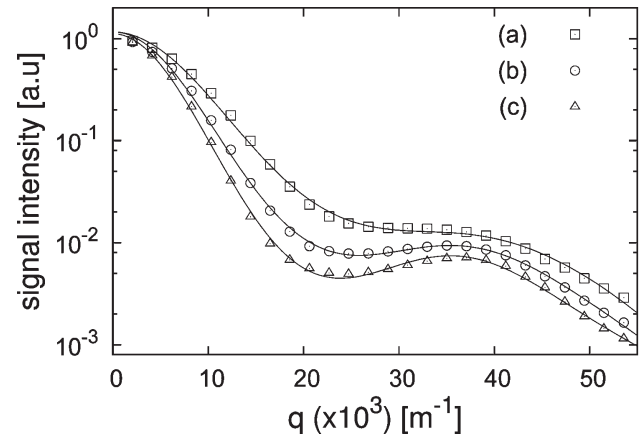

lattice of hexagons. In the current results, even though the water is now the continuous phase, a similar relationship seems to exist. The ratio between the positions of the two maxima for both oil in water emulsions in Figure 5 is very close to $\sqrt{ } 3$, giving an effective radius of the oil droplets for the two emulsions of $15 \pm 1$ and $25 \pm 2 \mu \mathrm{m}$.

Pore Hopping Analysis. The diffusion decay of the water around the oil droplets can also be analyzed using a pore hopping theory introduced by Callaghan et al. ${ }^{18}$ With this analysis, the average distance, $b$, between pools of intersticial water droplets can be calculated, along with the diameter $a$ of the water pools and the effective self-diffusion coefficient, $D_{\text {eff }}$, of the water. In order to test this approach, experiments were first performed on a standard sample of $30 \mu \mathrm{m}$ diameter polystyrene beads in water at three different diffusion observation times. The results of the fit for the three diffusion observation times are shown in Figure 6 (left). The values obtained from the fit are given in Table 2. Both diffusion observation times of 300 and $400 \mathrm{~ms}$ give good values for the inter pore spacing and average pore size. The diffusion observation time for $200 \mathrm{~ms}$ produces a value for the inter pore spacing that is a little too low. This is most probably due to the fact that the diffusion observation time is too small for the water to have had the opportunity on average to diffuse from one interstitial droplet to another.

The oil in water emulsion was the same as that used for the analysis in Figure 3, where the diameter was found to be 36.6 and $33.6 \mu \mathrm{m}$, respectively, from the particle sizing apparatus and from NMR using the analysis outlined in the previous section. The results of the fit to the pore hopping analysis are plotted in Figure 6 (right) and summarized in Table 2. From the figure, the fit is very good, comparable to the monodispersed polystyrene beads. The values for water droplet separation in the pore glass model are also in good agreement with the other methods though

Table 2. Summary of Pore Hopping Analysis on $30 \mu \mathrm{m}$ Diameter Monodisperse Polystyrene Beads and Monodisperse Oil in Water Emulsion of Diameter Approximately $36 \mu \mathrm{m}^{a}$

\begin{tabular}{lccccc}
\hline \multirow{4}{*}{ beads } & $\Delta[\mathrm{ms}]$ & $b[\mu \mathrm{m}]$ & $a[\mu \mathrm{m}]$ & $\xi[\mu \mathrm{m}]$ & $D\left[\mathrm{~m}^{2} \mathrm{~s}^{-1}\right]$ \\
\hline \multirow{6}{*}{ emulsion } & 200 & 25.8 & 10.1 & 4.9 & $2.02 \times 10^{-9}$ \\
& 300 & 29.3 & 9.8 & 4.3 & $1.98 \times 10^{-9}$ \\
& 400 & 31.0 & 9.4 & 3.9 & $1.81 \times 10^{-9}$ \\
& 200 & 35.3 & 10.3 & & $1.73 \times 10^{-9}$ \\
& 300 & 38.2 & 9.9 & & $1.78 \times 10^{-9}$ \\
& 400 & 39.6 & 9.7 & & $1.72 \times 10^{-9}$
\end{tabular}

${ }^{a}$ Estimate of error for dimensions $a$ and $b$ given the known size of polystyrene beads is $\pm 0.5 \mu \mathrm{m}$. Values of $\xi$ absent for emulsion due to very low or negative values obtained during nonlinear least squares fit of data.

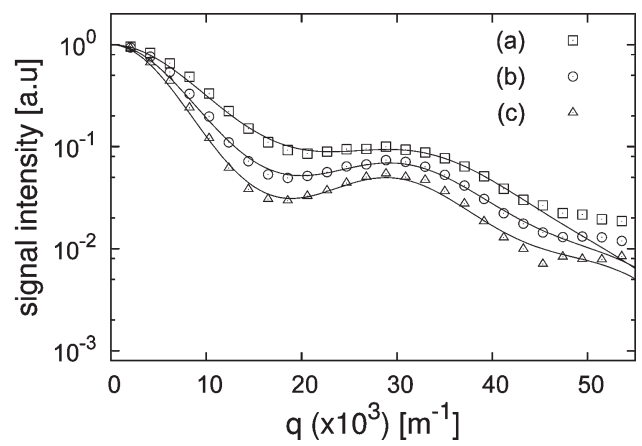

Figure 6. Fit of pore hopping model of Callaghan et al. ${ }^{18}$ to a monodisperse sample of polystyrene beads of $30 \mu$ m diameter in water (left) and a monodisperse oil in water emulsion of diameter $36.6 \mu \mathrm{m}$ (right). The different plots correspond to diffusion observation times of (a) $200 \mathrm{~ms}$, (b) $300 \mathrm{~ms}$, and (c) $400 \mathrm{~ms}$. 
slightly higher for the diffusion observation times of 300 and $400 \mathrm{~ms}$ at $39 \mu \mathrm{m}$. The droplet radius is comparable to that found for the polystyrene beads at $10 \mu \mathrm{m}$; however, the values for the distribution, $\xi$, were very low $\left(<10^{-9}\right)$ and depending on the fit sometimes negative. The pore glass model seems to also hold for the monodisperse oil in water emulsion. It was found that the diffusion observation time had to be chosen carefully in the experiment. Values greater than $500 \mathrm{~ms}$ gave experimental data that could not be fit using this model. There are probably a number of reasons for this: the simple form factor of a sphere used in the simulation as pointed out in the original paper ${ }^{17}$ may not be realistic, the diffusion observation length becoming greater than the characteristic diffusion length scale of the first shell of droplets, and the distortion of the shape of the oil droplets from simple spheres in the concentrated emulsion.

Oil Diffusion in the Emulsion. The mean squared displacement, $\left\langle z^{2}\right\rangle$, for the case of the water in the above example is expected to be $3.68 \times 10^{-9} \mathrm{~m}^{2}$ for a diffusion observation time of $400 \mathrm{~ms}$ given the free self-diffusion coefficient for water at $25^{\circ} \mathrm{C}$ is $2.3 \times 10^{-9} \mathrm{~m}^{2} \mathrm{~s}^{-1}$. This gives a sampling distance of $42 \mu \mathrm{m}$. The optimum diffusion observation time, $\Delta$, is given by $\Delta \approx b^{2} / 2 D_{\text {eff. }}{ }^{17}$ For the emulsion, this gives a value of approximately $450 \mathrm{~ms}$ and for the polystyrene beads $265 \mathrm{~ms}$. For the system under discussion, namely, $1 \%$ HMD in MCT, the self-diffusion coefficients of MCT and HMD are $3 \times 10^{-11}$ and $8 \times 10^{-11} \mathrm{~m}^{2} \mathrm{~s}^{-1}$, respectively, giving sampling distances of 5 and $8 \mu \mathrm{m}$, less than half the diameter of the smallest droplets attainable in this study. If the above equation holds, then the diffusion observation time would have to be set to approximately 26 and 10 s for the oil and HMD to observe diffraction peaks. Given the spin-lattice relaxation times of the two molecules, it would be very difficult to observe maxima in the diffusion response. However, information on the size of the oil droplets can be obtained from the diffusion response of the oil and tracer molecules within the oil as a function of diffusion observation time by fitting the response to the Murday and Cotts eq 2 or by calculating $\left\langle z^{2}\right\rangle$ at long diffusion observation times. Both methods rely on the fact that the oil or tracer remains within the same oil droplet during the diffusion observation time and cannot diffuse between droplets.

In Figure 7, the results are shown of the calculated $\left\langle z^{2}\right\rangle$ for MCT oil in an emulsion (a) and gel phase (c) and for HMD (b) in the emulsion only. In the emulsion, the $\left\langle z^{2}\right\rangle$ for both the MCT and HMD reach a plateau at long diffusion observation times $(>4 \mathrm{~s})$ showing that both do not diffuse beyond the oil-water interface of the droplets. The plateau values of the $\left\langle z^{2}\right\rangle$ for the MCT and HMD are $6.27 \times 10^{-11} \pm 4.9 \times 10^{-12}$ and $6.41 \times 10^{-11} \pm 3.2 \times$ $10^{-12} \mathrm{~m}^{2}$, respectively. An estimate of the mean and standard deviation is obtained from the values at times greater than $3.7 \mathrm{~s}$. They are in good agreement with one another. Using eq 5 , an estimate of the radius of the droplets can be made, and values of $12.5 \pm 0.5$ and $12.7 \pm 0.3 \mu \mathrm{m}$ are obtained for the MCT and HMD, respectively. The radius values compare well with the estimate obtained from the water diffraction experiments given in Figure 5. For the gel, at long diffusion observation times, the $\left\langle z^{2}\right\rangle$ of the HMD does not reach a plateau but increases linearly at long diffusion observation times to give an effective diffusion coefficient for the oil that is dependent on the membrane spacing in the gel and the permeability. ${ }^{20}$

The size of the monodisperse oil droplets can also be obtained from a fit of the echo decay of the MCT or HMD tracer using the Murday and Cotts equation. Figure 8 shows the values obtained for the radius of an emulsion as a function of diffusion observation time. At small diffusion observation times, the values obtained for the radius are prone to error and do not give the

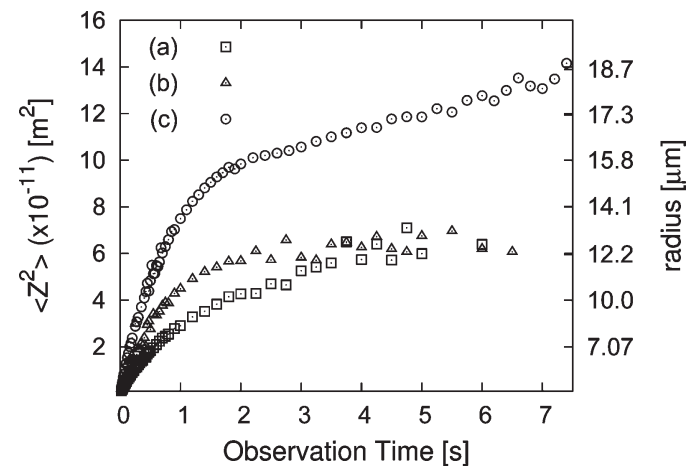

Figure 7. Mean squared displacement $\left\langle z^{2}\right\rangle$ of oil (MCT) and hexamethyldisilane (HMD) in a concentrated oil in water emulsion: (a) oil and (b) HMD. The average long time plateau value gives $\left\langle z^{2}\right\rangle$ of $6.27 \times 10^{-11} \pm 4.9 \times 10^{-12} \mathrm{~m}^{2}$ for the MCT oil and $6.41 \times 10^{-11} \pm 3.2 \times 10^{-12} \mathrm{~m}^{2}$ for the HMD. The average calculated radius for the droplets from MCT and HMD is then $12.5 \pm 0.5$ and $12.7 \pm 0.3 \mu \mathrm{m}$, respectively. Also plotted is the mean squared displacement of the HMD in a gel system generated from the same emulsion (c), where the water has been removed. At long diffusion observation times, the mean squared displacement increases at a constant rate, giving information on the permeability of the gel.

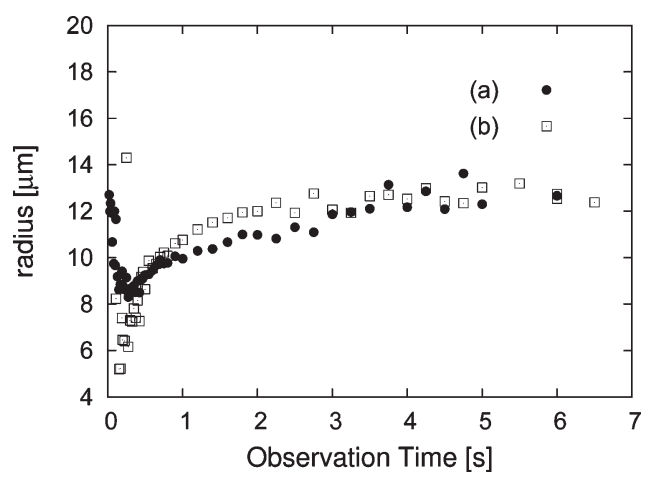

Figure 8. Calculation of the radius of the monodispersed oil in water emulsion using the equation derived by Murday and Cotts: ${ }^{10}$ (a) MCT oil, average value for the radius $=12.7 \pm 0.6 \mu \mathrm{m}$; (b) HMD tracer, average value for the radius $=12.7 \pm 0.3 \mu \mathrm{m}$.

correct value for the radius. It is only after a diffusion observation time greater than around $3 \mathrm{~s}$ that the values obtained seem to give a reasonable value. The final average values from 4 to $6.5 \mathrm{~s}$ was $12.7 \pm 0.6 \mu \mathrm{m}$ for the MCT and $12.7 \pm 0.3 \mu \mathrm{m}$ for the HMD. The Murday and Cotts equation is based on a number of assumptions. Not least is that the phase distribution that forms the experimental echo has a Gaussian distribution. This has consequences on the experimental conditions under which the model is valid. Below a certain diffusion observation time, not all molecules will have felt the effects of the restricting geometry and the model is invalid. This minimum time is given by the following inequality, $D \Delta / r^{2} \leq 1{ }^{26,27}$ For the experiments performed here, with a value for the free diffusion of oil $D=3 \times 10^{-11} \mathrm{~m}^{2} \mathrm{~s}^{-1}$ and radius $r=$ $12.5 \mu \mathrm{m}$, the minimum diffusion observation time is close to $5 \mathrm{~s}$, and for the HMD with a free self-diffusion coefficient $D=8.0 \times$ $10^{-11} \mathrm{~m}^{2} \mathrm{~s}^{-1}$, the diffusion observation time is close to $2 \mathrm{~s}$. Both values agree well with the experimental results shown in Figure 8 .

(26) Hindmarsh, J. P.; Su, J.; Flanagan, J.; Sing, H. Langmuir 2005, 21, $9076-$ 9084

(27) Hollingsworth, K. G.; Johns, M. L. J. Colloid Interface Sci. 2003, 258, 383-389. 


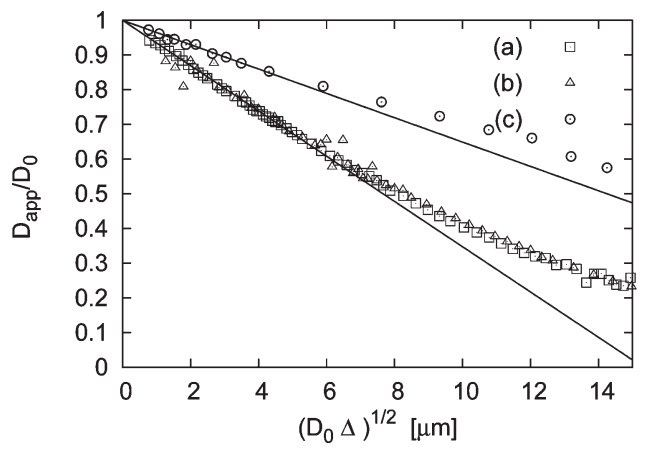

Figure 9. Diffusion response of MCT oil (a) and HMD (b) in a gel system at short diffusion observation times to estimate the surface to volume ratio of the gel network arising from the small diameter emulsion. $D_{0}$ for the MCT oil and HMD taken as $3.1 \times 10^{-11}$ and $8.0 \times 10^{-11} \mathrm{~m}^{2} \mathrm{~s}^{-1}$, respectively, to give an $S / V$ ratio of $3 / 11.4 \times$ $10^{-6} \mathrm{~m}^{-1}$. (c) Oil diffusion in gel arising from large droplet emulsion. $D_{0}$ for oil taken to be $2.9 \times 10^{-11} \mathrm{~m}^{2} \mathrm{~s}^{-1}$. The value of $S / V$ from the fit was found to be $3 / 21.4 \times 10^{-6} \mathrm{~m}^{-1}$.

Short Time Diffusion of Oil in the Gel: Surface to Volume. The value of the apparent self-diffusion coefficient $\left(D_{\text {app }}\right)$ in a porous material measured by pulsed-field gradient NMR even at short diffusion observation times is affected by the boundaries of the pores. In a series of papers by Mitra and co-workers, ${ }^{15,28-30}$ the dependence of $D_{\text {app }}$ versus diffusion observation time has been shown to be linearly related to the surface to volume ratio of the confining pore system and is given by eq 6 . A number of groups have applied this equation to measure surface to volume ratios in various porous media, ${ }^{21,29,31}$ and in a number of cases the approach has been validated using complementary methods ${ }^{32}$ or known data. ${ }^{21}$ The approach has not only been used with liquids as the probe molecule but also gases. ${ }^{33}$ The exact form of the equation is dependent on the pulse sequence used; however, it was found that, for the pore sizes and diffusion coefficients dealt with in this paper, that the correction terms are not significant and eq 6 was used as written when fitting the experimental data.

Figure 9 shows the results for two different gel systems prepared from two monodispersed oil in water emulsions of different diameter. The diameters of the starting emulsions were 25 and $46 \mu \mathrm{m}$. For the gel system made from the smaller diameter emulsion, both MCT (a) and HMD (b) data are shown. While for the gel made from the emulsion with the larger oil droplet diameter, only data from the MCT (c) is shown. Using values of $D_{0}$ of $3.0 \times 10^{-11}$ and $8 \times 10^{-11} \mathrm{~m}^{2} \mathrm{~s}^{-1}$ for the MCT oil and HMD, respectively, one observes that for the data from the gel prepared from the emulsion with the smaller droplet diameter that the experimental data overlap at short observations times to give the same slope. From this slope and eq 6, one can obtain a single value for the gel surface to volume ratio of $2.6 \pm 0.5 \times 10^{5} \mathrm{~m}^{-1}$ for the gel made from the smaller droplet emulsion. For the second gel, made from the emulsion of larger droplets, the surface to volume ratio was found to be $1.4 \pm 0.03 \times 10^{5} \mathrm{~m}^{-1}$. The trend in the $S / V$ ratios for the two gels is consistent with the initial size of the precursor emulsions. Since both gels are made from monodispersed emulsions, one can go a step further with the analysis of the $S / V$ values and approximate the polyhedral gel structure to

(28) Mitra, P. P Phys A (Amsterdam Neth) 1997, 241, 122-127.

(29) Mitra, P. P.; Sen, P. N. Phys. Rev. B 1992, 45, 143-156.

(30) Mitra, P. P.; Sen, P. N.; Schwartz, L. M. Phys. Rev. B 1993, 47, 8565-8574

(31) Zielinski, L. J.; Sen, P. N. J. Magn. Reson. 2003, 165, 153-161.

(32) Davies, C. J.; Griffith, J. D.; Gladden, A. J. S. L. F.; Johns, M. L. J. Magn. Reson. 2007, 187, 170-175.

(33) Mair, R. W.; Cory, D. G.; Peled, S.; Tseng, C. H.; Patz, S.; Walsworth, R. L. J. Magn. Reson. 1998, 135, 478-486. spheres and extract from them a characteristic radius from the relationship $S / V=3 / r$. The radius values obtained for the two gels by this treatment are $11.4 \pm 0.5$ and $21.4 \pm 0.5 \mu \mathrm{m}$, in good agreement to the initial size of the emulsions used during the preparation.

The transition from an emulsion to a polyhedral gel leads to an increase in the surface area of the system. For a monodisperse system, the increase in surface area is by a factor of 1.1 for the transition from a sphere to a space-filling Kelvin cell. ${ }^{6,34,35}$ An attempt was made, using the PGSTE sequence, to measure the surface to volume ratio of the two emulsions that were used to prepare the gels. Unfortunately, analysis of the results did not give the expected values for the surface to volume ratios given the known diameter of the droplets. It is thought that the main reasons for this were the sensitivity of the experiment to the size of the droplets used, the systematic errors introduced by the spectrometer, and the pulse sequence itself. It is known that if internal gradients are present, they can interfere with the simple PGSTE sequence. In emulsions, internal gradients arise at the interfaces between the oil and water due to the geometry of the interface and the natural differences in magnetic susceptibility of the oil and water. These internal gradients, which will affect only the dispersed (water) phase of the emulsion, if the droplets in the highly concentrated emulsion are still spherical, can interfere with the diffusion pulse sequence if they are large compared to the gradients applied during the pulse sequence, or if they change in size or polarity due to molecules diffusing in and out of regions where the internal gradients are not constant during the pulse sequence.

Although the internal gradients present in the emulsion are small, between 5 and $10 \mathrm{G} / \mathrm{cm}$ estimated from the half-height line width of the water resonance ${ }^{36,37}$ and the good agreement of the results of the other experiments with the expected values, it would appear that they cannot be discounted for the surface to volume experiment. In such experiments, it is necessary that the data are acquired by applying pulse gradients that are small. Standard implementations of the bipolar PGSTE sequences ${ }^{38}$ that are purported to compensate for the presence of static field gradients were tried with the emulsions and gels but without success. In recent years, further pulse sequences have been introduced that are much more robust and can compensate for the artifacts arising from the presence of internal gradients. ${ }^{39-41}$ In this study, such pulse sequences were not readily available. In the gel systems, the resonance lines in the spectra are much narrower than those in the spectra of the emulsions due to the removal of the water. Therefore, the data collected do not suffer to the same degree in terms of artifacts as the experiments with the emulsions. The results are more consistent with the expected values given the size of the initial emulsion droplets.

Taking values for the radius size of the emulsions obtained from other types of NMR experiments. The measured radii for the two emulsions were found to be $12.5 \pm 0.5$ and $23 \pm 0.5 \mu \mathrm{m}$. The surface to volume ratios were then $2.4 \pm 0.1 \times 10^{5}$ and $1.3 \pm$ $0.03 \times 10^{5} \mathrm{~m}^{-1}$ for the small and large drop emulsions, respectively. The ratio of the surface areas between the emulsion and gel

(34) Weaire, D.; Phelan, R. Philos. Mag. Lett. 1994, 70, 345-350.

(35) Weaire, D.; Phelan, R. Philos. Mag. Lett. 1994, 69, 107-110.

(36) Seland, J. G.; Sørland, G. H.; Zick, K.; Hafskjold, B. J. Magn. Reson. 2000, 146, 14-19.

(37) Drain, L. E. Proc. Phys. Soc., London 1962, 80, 1380-1382.

(38) Cotts, R. M.; Hoch, M. J. R.; Sun, T.; Marker, J. T. J. Magn. Reson. 1989, 83, 252-266.

(39) Zheng, G.; Price, W. S. J. Magn. Reson. 2008, 195, 40-44

(40) Galvosas, P.; Stallmach, F.; Kärger, J. J. Magn. Reson. 2004, 166, 164-173.

(41) Sun, P. Z. J. Magn. Reson. 2007, 187, 177-183. 


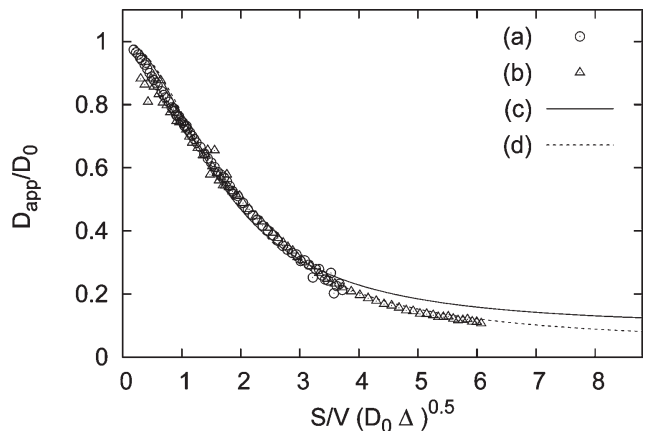

Figure 10. Normalized diffusion response of MCT oil (a) and HMD (b) in a gel system as a function of diffusion distance normalized by the surface to volume ratio. In both cases, the long time diffusion limit has not quite been reached. Two point Padé fit using eq 12 to MCT (c) and HMD (d) to give the tortuosity. $D_{0}$ for the oil and HMD taken as $3.0 \times 10^{-11}$ and $8.0 \times 10^{-11} \mathrm{~m}^{2} \mathrm{~s}^{-1}$ respectively. $S / V$ taken as $2.4 \times 10^{5} \mathrm{~m}^{-1}$.

system for the two cases were $1.1 \pm 0.45$ and $1.08 \pm 0.05$ for the small and large systems, respectively. These two values are in very good agreement with the expected theoretical value of 1.1. The large error for the gel prepared from the small droplets arises from estimating the error by propagating the error through from the an estimation of the radius of the emulsion. The good agreement of the mean value would suggest that during the preparation of the gel from the monodispersed emulsions there is very little coalescence of droplets to form larger structures and that the barrier properties of the three-dimensional gel formed will be strongly governed by the size of the initial emulsion.

Long Time Diffusion of Oil in the Gel. Figure 10 shows the apparent diffusion of the oil and HMD in the gel derived from the oil in water emulsion of approximate radius $12.5 \mu \mathrm{m}$. Both the tracer HMD and MCT oil follow the same diffusion response within the gel as a function of diffusion observation time. Even after several seconds, from this representation of the data, the apparent diffusion coefficient has yet to reach the asymptotic $D_{\infty}$ value. The dotted and full lines in the figure represent the best fit of the Padé approximation using eq 12. The values obtained for $1 / \alpha$ and therefore $D_{\text {app }} / D_{0}$ for the MCT and HMD were 0.093 and 0.043 , respectively. Figure 11 shows the same experimental data plotted in terms of mean squared displacement. In this plot, the data would suggest, at least for the HMD, an asymptote has been reached. A fit of the data, for points acquired with a $\Delta$ value greater than $2.6 \mathrm{~s}$, gives an average apparent diffusion coefficient of $1.83 \times 10^{-12} \mathrm{~m}^{2} \mathrm{~s}^{-1}$ for the HMD. For the MCT, a fit to data points acquired after $4 \mathrm{~s}$ gives a $D_{\text {app }}$ value of $3.44 \times 10^{-12} \mathrm{~m}^{2} \mathrm{~s}^{-1}$. This leads to $D_{\infty} / D_{0}$ values of 0.063 and 0.043 for the MCT and HMD, respectively. The two point Padé approximation does not give a particularly good fit over the full data range. However, it is useful in giving an estimate for the lower bound for $D_{\text {app }} / D_{0}$, while the analysis of the data as shown in Figure 11 gives an estimate of the upper bound. Therefore, a conservative estimate for the range of final $D_{\infty} / D_{0}$ values for the HMD and MCT oil in the gel system would be that they lie between 0.1 and 0.04 .

Since both MCT oil and HMD reach the same fractional infinite diffusion value, the permeability of the two oils are solely

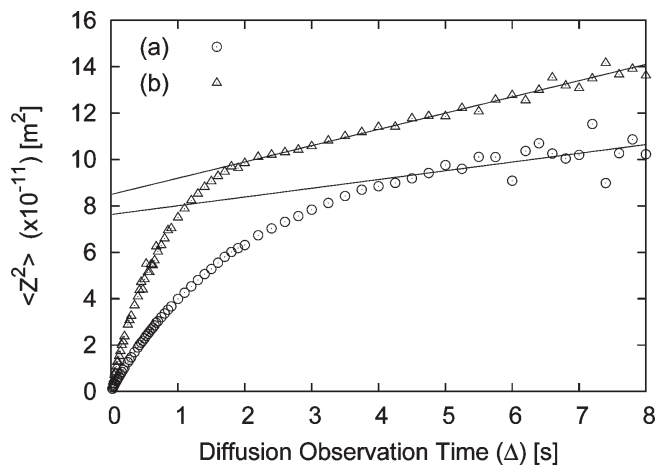

Figure 11. Mean squared displacement of MCT oil (a) and HMD (b) in gel a system as a function of diffusion observation time. The two straight lines are from fitting the linear parts of the curve to $\left\langle z^{2}\right\rangle=2 D \Delta+c$.

dependent on their free diffusion coefficient value and the separation of the protein gel barriers, which in this case is proportional to the radius value obtained from the short time surface to volume ratios. The permeability values obtained for the MCT oil lie between $1.2 \times 10^{-7}$ and $4.8 \times 10^{-8} \mathrm{~m} \mathrm{~s}^{-1}$ and for the HMD $3.2 \times 10^{-7}$ and $1.3 \times 10^{-7} \mathrm{~m} \mathrm{~s}^{-1}$. Such values lie in the range observed for the permeability of water in a water in oil in water double emulsion, $1.43 \times 10^{-6} \mathrm{~m} \mathrm{~s}^{-1}$, as measured by selfdiffusion $\mathrm{NMR}^{26}$ and for the low molecular weight fluorescent molecule 5(6)-carboxyfluorescein $\left(6.2 \times 10^{-8} \mathrm{~m} \mathrm{~s}^{-1}\right)$ across the aqueous interface of a semipermeable nanoparticle colloidosome system. ${ }^{42}$ Therefore, the permeability of the protein gel network should have some interesting applications in the area of encapsulation.

\section{Conclusions}

The work discussed demonstrates the utility of preparing emulsions with a controlled and known size distribution so that their physical properties can be more easily determined. The physical and dynamic properties of the emulsion could be characterized simply and interpreted without ambiguity using a variety of self-diffusion NMR techniques. In the emulsion system, the size and arrangement of the droplets could be determined together with the permeability of the interface. On going from an emulsion to a gel system, the internal structure of the gel system was probed and the changes in dimensions agreed well with theory. Finally, the permeability of the protein network in the gel system to the oil and probe molecules could be estimated using a simple model based on the regular dimensions of the network, and it was shown that the interface had good permeability properties for possible use in encapsulation in food related areas.

Acknowledgment. The authors would like to thank Nestec Ltd for time and permission to publish the work and Dr. Adam Burbidge for assistance in proofreading the document and technical discussions.

(42) Lee, D.; Weitz, D. A. Adv. Mater. 2008, 20, 3498-3503. 